\title{
Flexible hybrid WDM/TDM PON architectures using wavelength selective switches
}

\author{
Goutam Das, Bart Lannoo, Abhishek Dixit, Didier Colle, Mario Pickavet, Piet Demeester \\ Ghent University - IBBT, Gaston Crommenlaan 8, B-9050 Gent, Belgium \\ Phone: +3293314970 \\ Fax: +3293314899 \\ Email: goutam.das@intec.ugent.be \\ Website: http://www.ibcn.intec.ugent.be
}

\begin{abstract}
For building an optical access network, we propose some new hybrid WDM/TDM passive optical network (PON) architectures that use wavelength selective switches (WSSs) at the remote node to improve flexibility, data security and power budget. Through simulations we demonstrate that the switching capabilities of a WSS can provide additional gains in terms of wavelength usage by a better statistical multiplexing. Several WSS-based hybrid WDM/TDM PON variants are proposed and assessed. These architectures are also compared with the more commonly used hybrid WDM/TDM PONs consisting of power splitters and/or arrayed wavelength gratings (AWGs), in terms of cost and power budget.
\end{abstract}

Keywords: optical access; PON architectures; hybrid WDM/TDM; flexibility; dynamic bandwidth allocation

\section{Introduction}

Time division multiplexing (TDM) passive optical networks (PONs), like Ethernet PON (EPON) and Gigabit PON (GPON), are now widely accepted as optical access network solutions to distribute reasonably high bandwidths to the customers through an optical fiber network infrastructure [1-3]. However, due to the advent of bandwidth extensive services like high-definition video or interactive gaming, wavelength division multiplexing (WDM) PONs are introduced to increase the capacity for each individual user. As a pure WDM PON tends to waste bandwidth due to a lack of flexibility, hybrid WDM/TDM PON architectures are proposed in the literature with different ranges of flexibility [4-5]. A hybrid WDM/TDM PON combines the WDM domain with a TDM PON and can deliver both the benefits of an increased capacity delivered by WDM and the inherent capacity sharing and flexibility of a TDM PON. Note that we refer to 'flexibility' as the architectural capability to offer the same network (PON) performance with a smaller number of used wavelengths.

One of the main hybrid WDM/TDM PON flavors is a broadcast and select $(B \& S)$ PON architecture that uses passive power splitters to broadcast all the wavelengths to all the users, and leaves it to the media access control (MAC) layer to schedule time slots as well as wavelengths to different users. It requires tunability at the optical network unit (ONU) at the 
customer premises. Due to the overall broadcast nature of this architecture it has serious security issues and provides no data isolation whatsoever. It is also easy to see that as the number of users per B\&S-PON increases, due to the high fan-out of the passive splitting stage, it has a poor power budget and therefore restricts the overall reach. Operators today, however, prefer to have a higher degree of node consolidation (i.e., more users covered by a single PON infrastructure) that covers a larger geographical area (long reach PON) [6]. Therefore, a B\&S-PON might not be the most suitable candidate even though it offers the highest flexibility in terms of bandwidth utilization. Another important hybrid WDM/TDM PON architecture, referred to as a wavelength splitting (WS) PON, uses a combination of wavelength splitters (e.g., arrayed waveguide grating, AWG) and power splitters to share each wavelength among multiple ONUs in the time domain. Though this architecture significantly improves the data security and the power budget, it reduces the overall flexibility available in a B\&S-PON. Besides these two major hybrid WDM/TDM PON candidates, B\&S-PON and WS-PON, other architectures are proposed in the literature. One of these proposals uses a combination of power splitters and optical switches (based on semiconductor optical amplifier, SOA) [7-9], and is referred to as active routing optical access network (ARON) [78] or wavelength routed (WR) PON [9]. A WR-PON uses power splitters to distribute all the wavelengths to every user. Additionally it uses a SOA switch bank to control and restrict the number of wavelengths delivered to a group of end users according to their need. Therefore, a WR-PON suffers from the same power budget issue as a B\&S-PON. In addition, it uses active components at the remote node that breaks away from the passive nature of the PON philosophy. However, due to its switching functionality it improves the data security without compromising flexibility.

In this paper, we propose a novel scheme to incorporate the good points of the three solutions mentioned above and to avoid the hind side of each. In particular, we propose to use wavelength selective switches (WSS) with different configurations. This might increase the overall cost of the proposed architecture due to the expensive WSS as well as the requirement for power provisioning in presence of active components at the remote node. However, we will prove from our cost analysis that the introduction of WSS does not affect the cost per customer significantly due to the higher reach, wavelength sharing and added flexibility. The rest of this paper is organized as follows. In section 2, we provide a brief description of the general hybrid WDM/TDM PON architecture and introduce an architecture classification according to three levels of flexibility, ranging from fully flexible, over partially flexible to fully static. Section 3 lists the benefits of architectural flexibility and its associated 
constraints. Section 4 demonstrates through simulation the benefits of a (partially) flexible architecture, and illustrates the additional flexibility gain by using a WSS-based architecture. Section 5 introduces several novel hybrid WDM/TDM PON architectures using a WSS as the key component. Section 6 provides a cost and power budget comparison for the different proposed WSS-based hybrid WDM/TDM PON architectures, benchmarked to the more common B\&S-PON and WS-PON architectures. Finally, section 7 concludes the paper.

\section{Hybrid WDM/TDM PON architectures}

This section presents a general hybrid WDM/TDM PON architecture consisting of two remote nodes, of which remote node 1 (RN1) is varied to introduce different architectural variations and levels of flexibility. Further, the hybrid WDM/TDM PON architectures are classified according to the flexibility of RN1 and special attention is given to partially flexible architectures.

\subsection{General hybrid WDM/TDM PON architecture}

A general architecture of a (flexible) hybrid WDM/TDM PON is shown in Figure 1, consisting of an optical line terminal (OLT) in the central office, an optical network unit (ONU) at the user side, and two remote nodes (RN). The OLT consists of $W_{u}$ uplink line cards and photo detectors (PD), and $W_{d}$ downlink line cards and distributed-feedback (DFB) lasers. All uplink/downlink wavelengths are (de)multiplexed by using e.g., arrayed waveguide gratings (AWGs) as wavelength splitter/combiner, and both wavelength bands are put on the same fiber by using a three-port circulator. The ONU contains a three-port circulator to separate the uplink and downlink wavelengths. Further, the ONU has an uplink line card and a tunable burst mode transmitter (Tx) for tuning to any desired wavelength, and a downlink line card, a PD and a tunable optical filter for selecting the desired wavelength. Note that with the current technologies, the realization of tunable optical filters with sharp notches, required for hybrid WDM/TDM PONs, can be very expensive, but our believe is that these devices will be available in the future for reasonable costs. By varying RN1 (1:M split), several hybrid WDM/TDM PON architectures are presented in this paper. In RN2, a passive power splitter (1: $N$ split) is installed, which means that RN1 is connected to $M$ TDM PONs. 


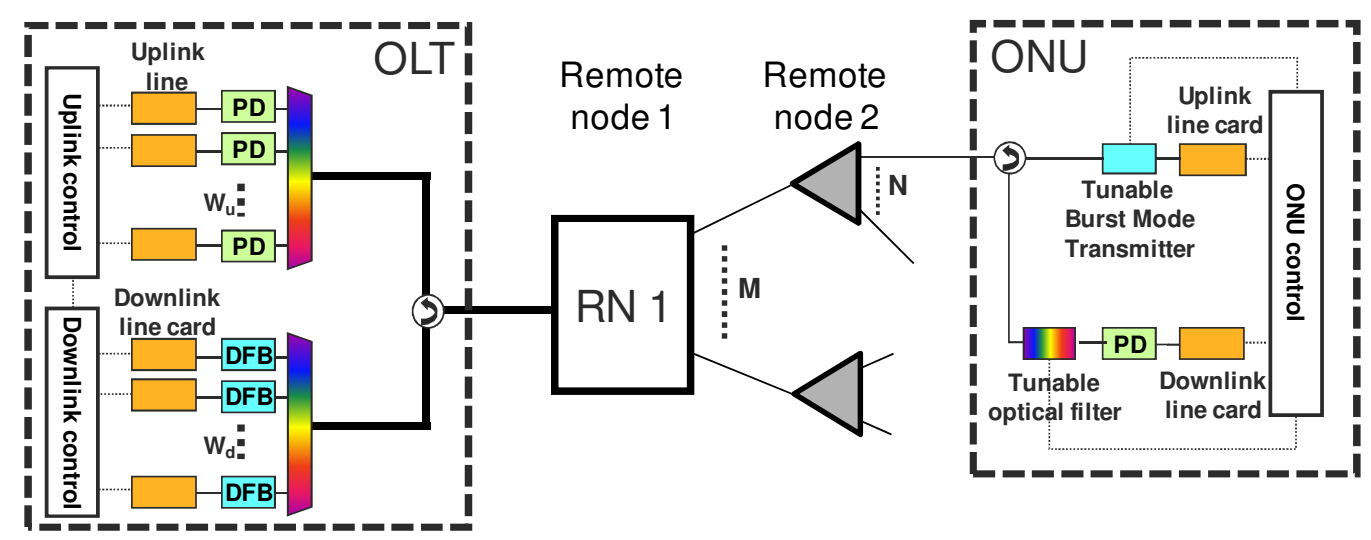

Figure 1: Hybrid WDM/TDM PON architecture, consisting of two remote nodes

\subsection{Hybrid WDM/TDM PON classification in terms of flexibility}

Based on the flexibility of RN1, the hybrid WDM/TDM PONs can be classified in three main categories: (1) fully flexible, (2) fully static and (3) partially flexible.

(1) In a fully flexible architecture, each wavelength can simultaneously be routed to any TDM PON (or RN2), and by consequence each TDM PON can be reached by any wavelength.

These architectures have an inherent broadcast facility, but most variants suffer from higher power losses due to the high insertion losses of optical power splitters. The already mentioned B\&S-PON and WR-PON fall under the category of a fully flexible architecture.

(2) In a fully static architecture, each wavelength is routed to only one fixed TDM PON (or RN2), and each TDM PON can be reached by only one fixed wavelength. Such architecture has a low component insertion loss and high security. The mentioned WS-PON, with only AWGs in RN1, is an example of a fully static architecture.

(3) In a partially flexible architecture, each TDM PON can be reached by multiple wavelengths. However, each wavelength can reach either multiple TDM PONs (multicasting flexibility), only one TDM PON independently of the other wavelengths (switching flexibility) or a combination of both (multicasting and switching flexibility), and for that reason, we make a distinction between these two types of flexibility.

* Multicasting flexibility refers to the flexibility where one wavelength can be shared by multiple TDM PONs, but not every TDM PON cannot be reached by that wavelength.

* Switching flexibility refers to the flexibility where one TDM PON can be independently reached by any wavelength, but one wavelength cannot reach multiple TDM PONs. 


\subsection{Partially flexible hybrid WDM/TDM PON architectures}

In general, a partially flexible architecture is more costly than a fully flexible or fully static one, but it has a higher security and lower component insertion loss than a fully flexible architecture, and is, of course, more flexible than a fully static one. In this way, it combines the strengths of a fully flexible and fully static architecture and often, a trade-off between cost, insertion loss, security and flexibility will decide about the best architectural variant in a specific situation.

The most straightforward partially flexible architecture consists of a combination of a B\&SPON and WS-PON, using a combination of AWGs and power splitters in RN1 (only offering multicasting flexibility). Replacing the AWG by a WSS can further improve the flexibility (offering multicasting and switching flexibility) without compromising in insertion loss or security issues [10]. We propose to use a WSS for offering switching flexibility as the insertion loss of a WSS does not increase with the port count in contrary to a power splitter. WSS are generally implemented in micro-electro-mechanical systems (MEMS) that provide low insertion loss wavelength switching capabilities [11]. Off-the-shelf WSS can have the functionality of $1 \times 2,1 \times 4$ or $1 \times 8$ switching. They are available with both $100 \mathrm{GHz}$ and $50 \mathrm{GHz}$ channel spacing, i.e. with 48 and 96 wavelengths channels, respectively. When it is used as reconfigurable optical demultiplexer, a WSS can steer each optical channel present on its common input port towards one of its output ports. On the other hand, in the reverse direction it can be used as wavelength blocking device, where it can block some of the wavelengths from each of the ports to enter to the common port. However, it can be configured in a manner that it will allow all the possible wavelengths from each of the output ports to enter into the common port.

Both partially flexible architectures are evaluated in section 4, proving the advantages of a partially flexible architecture and the extra flexibility gain delivered by a WSS. In section 5 we propose different additional architectural variations of RN1 using a WSS to provide different degrees of partial flexibility.

\section{Flexibility benefits and constraints}

In this section we briefly describe the benefits of adding flexibility in a hybrid WDM/TDM PON. This provides us the motivation for our flexibility enhancement proposals for the hybrid WDM/TDM PON architectures. Additionally, we also mention the constraints that come with an enhanced flexibility. 


\subsection{Flexibility benefits}

In a typical hybrid WDM/TDM PON, multiple TDM PONs can be set up from the OLT, each at a specific wavelength. Each TDM PON serves a set of users, and within this set, the capacity is shared. However, by means of wavelength selection or routing, the number of users within the set can be varied, and thus the capacity offered per user can be varied. Hence a flexible hybrid WDM/TDM PON can offer capacity-on-demand, and the congestion probability can be significantly reduced compared to a static wavelength configuration. In the next subsections, we give a brief account of the three most striking features of a flexible architecture that separate it from most of the other PON architectures, i.e., network extensibility, energy efficiency and network migration.

\section{Network extensibility}

Network extensibility refers to the efficient deployment strategies that the hybrid WDM/TDM PON architecture can promote while constantly expanding the network by the incremental installation of network equipment according to the end user demands. Due to the inherent flexibility, a flexible architecture can be used for green- or brown-field installations where the user demands can change and evolve over time. This provides a means for the operator for a smooth and incremental expansion of the network according to the demand, while keeping the basic network infrastructure deployed in the field undisturbed.

\section{Energy efficiency}

Our proposal for energy efficiency has a similar argument as provided for the network extensibility. The network operator can regroup the users when the overall network traffic demand is low in a manner that it can eventually switch off some of its active equipment. The flexibility of the hybrid PON architecture facilitates this whenever required remotely without touching the real infrastructure. This might help the operator to build its network greener. The same scheme also depicts the possibility of dynamic allocation of wavelengths amongst users according to their traffic needs.

\section{Network migration}

Our proposal for network migration corresponds to an efficient migration strategy that the flexible architecture can support during e.g. a technology upgrade. In this way, co-existence of a new technology with the legacy system is made possible. Due to the possibility of wavelength allocation on demand, the new and the legacy users can be grouped in different 
wavelengths and can co-exist seamlessly irrespective of their physical location and technological mismatch. This strategy allows a more smooth transition of the network during the migration phase.

\subsection{Constraints raised by enhanced flexibility}

Adding extra flexibility in the access network comes also at the cost of some drawbacks, which have to be carefully tackled. The most important constraints are equipment cost, power budget and security issues.

\section{$\underline{\text { Equipment cost }}$}

Introducing (active) equipment like a WSS will increase the total equipment cost. However, the network operation costs, e.g. power consumption, service provisioning, migration, maintenance, etc., should be decreased by an increasing degree of flexibility. E.g., the power consumption should be optimized by reconfiguring the network (as explained in the previous subsection), the service provisioning and migration might be remotely done, etc. This can reduce even the total cost of ownership (TCO) as TCO depends on both equipment costs as well as network operation costs. Of course, the equipment costs have to be kept under control to exploit the possible flexibility benefits in terms of TCO.

\section{Power budget}

The power budget specifies the total optical power required from the OLT to the ONUs, and will be highly affected by an increasing power splitting ratio. Limiting the power splitting ratio and decreasing the power budget is one of the main challenges tackled in this paper.

\section{$\underline{\text { Security issues }}$}

The security issues generally come from the broadcast or multicast nature of the PON infrastructure. Therefore, PONs with a higher multicasting domain suffer from more severe security threats due to the sharing of the same media by more ONUs (users). Adding flexibility might facilitate the operator to selectively choose the multicasting domain according to the traffic demand for minimizing the security threats.

\section{Evaluation of flexibility in hybrid WDM/TDM PONs}

As described in section 2, hybrid WDM/TDM PONs can have a different degree of flexibility. The more flexible architectures, however, are either more expensive, experience a higher 
insertion loss or are less secure. A question that arises is if a fully flexible architecture really needed, or if a partially flexible architecture already can serve the flexibility advantages listed in section 3, without compromising on the flexibility constraints. An important assessment parameter for flexibility is the number of wavelengths required at a certain network load. For a fully flexible architecture, it is clear that the number of wavelengths required can be optimally minimized, but often the gain of a fully flexible architecture compared to a partially flexible one, and the minimum degree of flexibility required to have a significant advantage is not straightforward. In this section, an evaluation is made for different degrees of flexibility of a hybrid WDM/TDM PON.

In section 2 we have also introduced two types of flexibility gain offered by partially flexible architectures, i.e., multicasting and switching flexibility. In this section we particularly evaluate through simulation two different architectural options for a hybrid WDM/TDM PON, in terms of wavelength usage: one architecture consisting of power splitters and AWGS (providing multiplexing gain), and another one consisting of power splitters and WSSs (providing both multiplexing and switching gain). As multicasting flexibility involves power splitters which increase the power budget, we prefer to replace multicasting flexibility by switching flexibility wherever possible, e.g. by using WSSs. This enhances the overall power budget of a PON infrastructure and increases its reach and node consolidation. In this section our aims are to assess the flexibility of hybrid WDM/TDM PONs and to prove how switching flexibility can replace multicasting flexibility. The next sub-section provides the simulation environment for our flexibility evaluation, then the two evaluated architectures are presented, and finally a detailed discussion is given about our key findings.

\subsection{Simulation setup for flexibility evaluation}

We have simulated a hybrid WDM/TDM PON with $W_{u, d}$ wavelengths available for each OLT and $M$ TDM PONs, each consisting of $N$ ONUs (corresponding to $M \times N$ ONUs in total), as depicted on Figure 1. For our simulations, we have chosen $M=16, N=4$ and $W_{u, d}=16$. From the access side, packets arrive at the ONU from a user connected to that ONU. Packets are buffered in the ONU until the ONU is allowed to transmit them to the OLT.

\section{Traffic source for generating highly bursty traffic}

In our model, we consider $R_{D}$ to be the data rate of the access link from a user to an ONU, and $R_{U}$ to be the date rate of an upstream channel from an ONU to the OLT. We have chosen, $R_{U}=1 \mathrm{~Gb} / \mathrm{s}$ and $R_{D}=R_{U} / N=250 \mathrm{Mb} / \mathrm{s}$. We have generated packets in the form of Ethernet 
frames (64 to1518 bytes) and packets arrive at each ONU from the end user. The simulated user traffic is self-similar. In [12], it is demonstrated that Ethernet local area network (LAN) traffic is statistically self-similar and none of the commonly used traffic models are able to capture this fractal behavior. Self-similarity is where a certain property of an object is preserved with respect to scaling in time and/or space. There are notable differences in the behavior of self-similar traffic with the traditional traffic models. There is no natural length of a "burst": at every time scale ranging from a few milliseconds to minutes and hours, similarlooking traffic bursts are evident and aggregating streams of such traffic typically intensifies the self-similarity ("burstiness") instead of smoothing it. It is now clear that such a behavior has serious implications in the design, control and analysis of networks. Self-similar traffic can be generated by multiplexing several sources of Pareto-distributed ON and OFF periods. Pareto distribution has the following probability density function:

$$
P(x)=\frac{a b^{\alpha}}{x^{\alpha+1}}, \quad x \geq b
$$

where $\alpha$ is a shape parameter (tail index), and $b$ is the minimum value of $x$. For $\alpha \geq 2$, the variance of the distribution is infinite and for $\alpha \leq 1$, the mean value is infinite as well. For self-similar traffic, $\alpha$ should be between 1 and 2. The value of $\alpha$ has been chosen according to the actual measurements on Ethernet traffic [12]. We generate the self-similar traffic by aggregating 32 sub-streams [13], each consisting of alternating Pareto-distributed ON/OFF periods (Figure 2), with $\alpha=1.4$ for the ON period and $\alpha=1.2$ for the OFF period. In the ON period, the packet arrivals are exponentially distributed with a mean arrival rate of $A_{r}(\mathrm{in} \mathrm{b} / \mathrm{s})$. The traffic load can be produced by variable values of $A_{r}$ and the location parameter for the $\mathrm{ON}$ and OFF period. Different levels of burstiness can be achieved by varying $A_{r}$. For the same load, an ONU will be on for less periods for a higher value of $A_{r}$, and the traffic will be more bursty. To produce highly bursty traffic for the simulations in the following section, we have used an $A_{r}$ value of $12.5 \mathrm{Mb} / \mathrm{s}$ for all loads. 


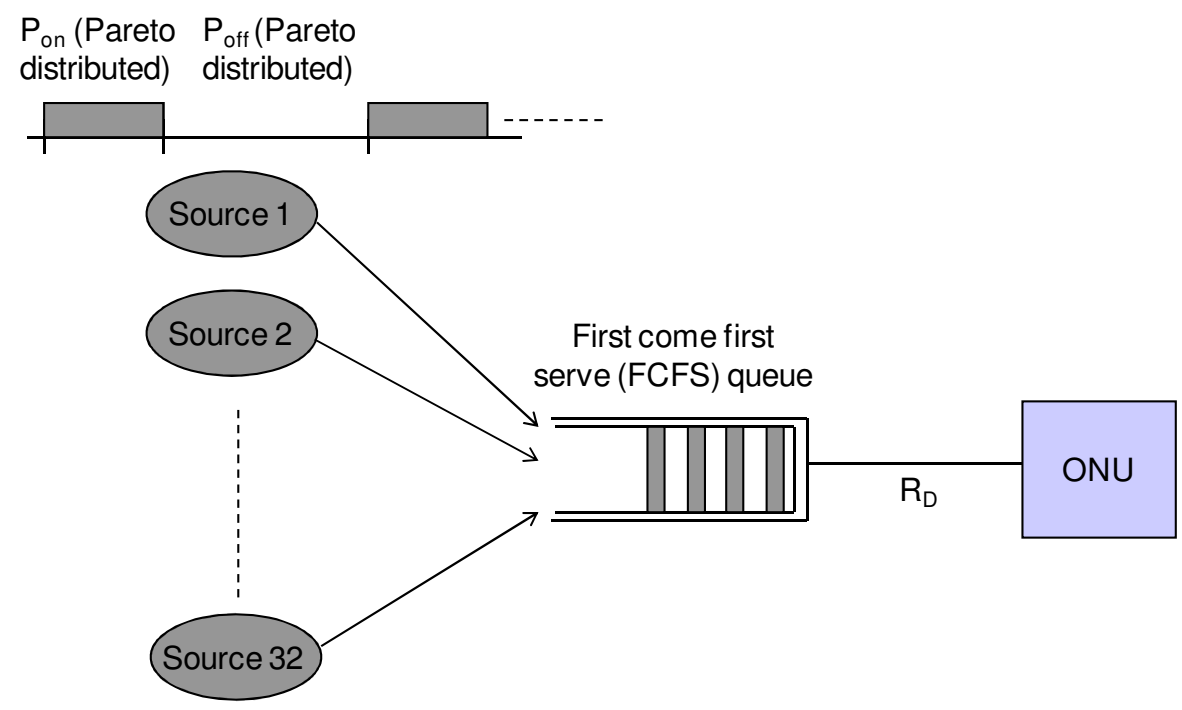

Figure 2: Self-similar traffic generation model

\section{Offline MAC protocol}

For evaluating different architectural options for a hybrid WDM/TDM PON, we need a medium access control (MAC) protocol [9] that exploits the offered flexibility in terms of dynamic wavelength allocation. To investigate the optimal degree of flexibility in hybrid WDM/TDM PON architectures, we have used an offline joint time and wavelength assignment scheme based MAC protocol [14-16]. The offline approach has very less implementation complexity and can address fairness issues among different ONUs, and can also be used for more advanced hybrid WDM/TDM PON architectures using remote optical switching. In addition to this, in offline algorithms the OLT would wait until the report messages from all ONUs have arrived and would then try to arrange upstream scheduling in an optimal way, thus minimizing void formation and wavelength switching, and maximizing wavelength usage.

Note that especially the WSS tuning time might be significantly high (e.g., $1 \mathrm{~ms}$ ), and therefore wavelength reshuffling in a frame by frame basis (i.e., wavelength allocation for ONUs might change from one MAC frame to another one) would require WSS to be switched in every frame. This might prove to be inefficient if a significant amount of time (and hence wavelength utilization) is lost due to WSS switching. However, as offline scheduling leaves an inter-frame scheduling gap (IFSG) between frames [9], [14], we can utilize this IFSG for performing the WSS switching. To keep the wavelength utilization high one might have to increase the cycle time which might have a detrimental effect on the quality of service (QoS) parameters like delay and jitter. However, the offline scheduling and the MAC protocol can 
be modified to improve the QoS with larger cycle time. The detailed discussion related to the QoS handling and the associated MAC improvement is out of scope of the current paper.

\subsection{Hybrid WDM/TDM PON architectural configurations}

The described simulation setup is used in the following subsections to evaluate the multicasting flexibility (by using power splitters) and switching flexibility (by using wavelength selective switches (WSS)) of a hybrid WDM/TDM PON.

\section{Architectural configuration to measure the influence of just multicasting flexibility}

For the multicasting flexibility evaluation, RN1 consists of an AWG followed by multiple ( $m_{A W G}$ for the uplink and downlink direction, respectively) power splitters, to distribute one wavelength to different TDM PONs, as shown in Figure 3(a). Here we have chosen a partially flexible architecture with AWGs and power splitters to distinguish the benefits of multicasting flexibility gain from the switching flexibility gain. As we still use power splitters along with AWGs, some multicasting flexibility is still available. Both the component insertion loss - and reach - and flexibility, however, decrease, when $m_{A W G}$ increases. The trade-off between insertion loss and flexibility defines the optimal choice of $m_{A W G}$.

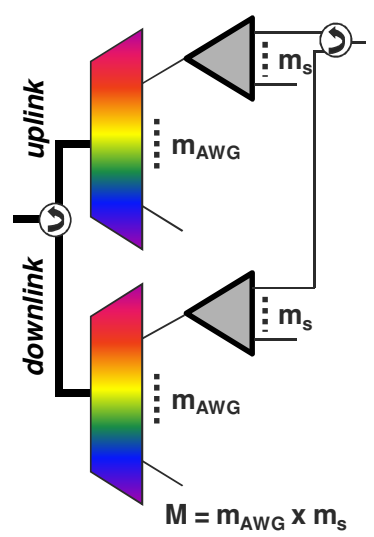

(a)

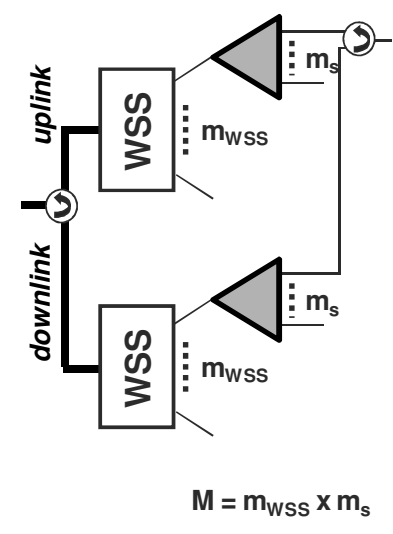

(b)

Figure 3: Architecture of RN1 in a partially flexible hybrid WDM/TDM PON using (a) AWGs and power splitters and (b) WSSs and power splitters

By varying the values for $m_{s}$ and $m_{A W G}$, while keeping $M\left(=m_{s} \times m_{A W G}=16\right)$ constant (as shown in Figure 3(a)), five different levels of flexibility in RN1 are evaluated. Group $m_{s}$ indicates the number of TDM PONs that can share the same wavelength, or the number of wavelengths that can be used by one TDM PON (or RN2). For two extreme cases, this 
architecture is reduced to a fully flexible broadcast-and-select architecture $\left(m_{A W G}=1\right)$ and a fully static wavelength-split architecture $\left(m_{s}=1\right)$, respectively. In the fully flexible architecture, any of the subscribers can use any of the 16 available wavelengths (Group16). In the fully static architecture, all ONUs can only use their dedicated wavelength (Group 1). The other architectures then represent the partially flexible architectures and specify that only 8,4 or 2 specific wavelengths (Group 8, 4,2) can be used by a TDM PON sharing the last mile passive splitter in RN2. Therefore, any wavelength can only be shared by a limited number (8, 4 or 2) of TDM PONs.

\section{Architectural configuration to measure the influence of switching flexibility}

For the switching flexibility evaluation, the static AWG in RN1 of Figure 3(a) is replaced by a reconfigurable WSS as shown Figure 3(b). This implies that any wavelength can be steered to any of the TDM PONs without any restriction. In this way, we can evaluate if extra gain can be reached due to the additional switching flexibility offered by a WSS. We have again assumed the same number of TDM PONs per group attached to the same multicasting domain. We have chosen this architecture for our analysis as it is very similar to the AWG based architecture of Figure 3(a) and hence facilitates a better comparison among them. All the other WSS-based architecture proposed in section 5 will have a similar implication as the considered architecture of Figure 3(b) and therefore a detailed analysis of their relative gain is left for a future evaluation. In this paper we restrict our flexibility analysis to the evaluation of the general benefits by introducing WSS elements in the hybrid WDM/TDM PON scenarios in place of AWGs, rather than quantifying the relative gains of all the WSS architectures proposed in section 5 .

\subsection{Simulation results and discussion}

To perform a common simulation for both architectures in Figure 3, we assume - for the purpose of our simulation - that all the individual TDM PON groups are no longer restricted by a maximum number of wavelengths per group. For our evaluation, we also relax the restriction for the overall number of wavelengths of 16, as this will enable us to study the wavelength demand without creating any additional packet delay or packet drop due to the unavailability of wavelengths for a particular TDM PON during the heavily loaded frames. According to our assumption, each group of TDM PONs is thus allocated as many wavelengths as it demands within a particular frame. In this way, the simulation scenario described above assumes full flexibility in terms of wavelength switching (so that any 
wavelength can be steered to any TDM PON) and partial flexibility in terms of multicasting that varies according to the group number specified previously (so that any of the wavelengths can be shared among all the TDM PONs within the specified group, e.g., four TDM PONs together for the Group 4 scenario). Therefore, the simulations carried out for the two architectures of Figure 3 are the same. It is only the interpretation of the results obtained (as will be shown later in this section) that discriminates between them. In the next subsection we will show the simulation results providing an estimation of the flexibility gains for both architectures shown in Figure 3(a) and (b).

\section{Flexibility gains offered by partial flexibility}

Figure 4 and 5 provide the plot for the average and the maximum number of wavelengths, respectively, required for the different overall traffic load (generated through our bursty traffic source) for each TDM PON for the unified and unrestricted simulation scenario. The plots are especially useful when we fix the number of wavelengths as shown by the horizontal line in Figure 4 and 5. The cross section with the wavelength curves for the different groups provides a similar delay and packet loss scenario for all groups. Therefore, it clearly shows that with a higher number of TDM PONs per group (i.e., more multicasting and switching flexibility), we get higher overall network throughput for the same delay and packet loss performance.

Figure 4 also illustrates that as the flexibility (group number) increases; the gain due to the added multicasting flexibility diminishes (cf. Group 8 vs. Group 16). Therefore, it suggests that a partially flexible network can be almost as good as a fully flexible network. Due to the highly bursty nature of the traffic, we also observe a large discrepancy between the average and the maximum number of wavelengths required. Therefore the wavelength requirement between frames varies significantly. 


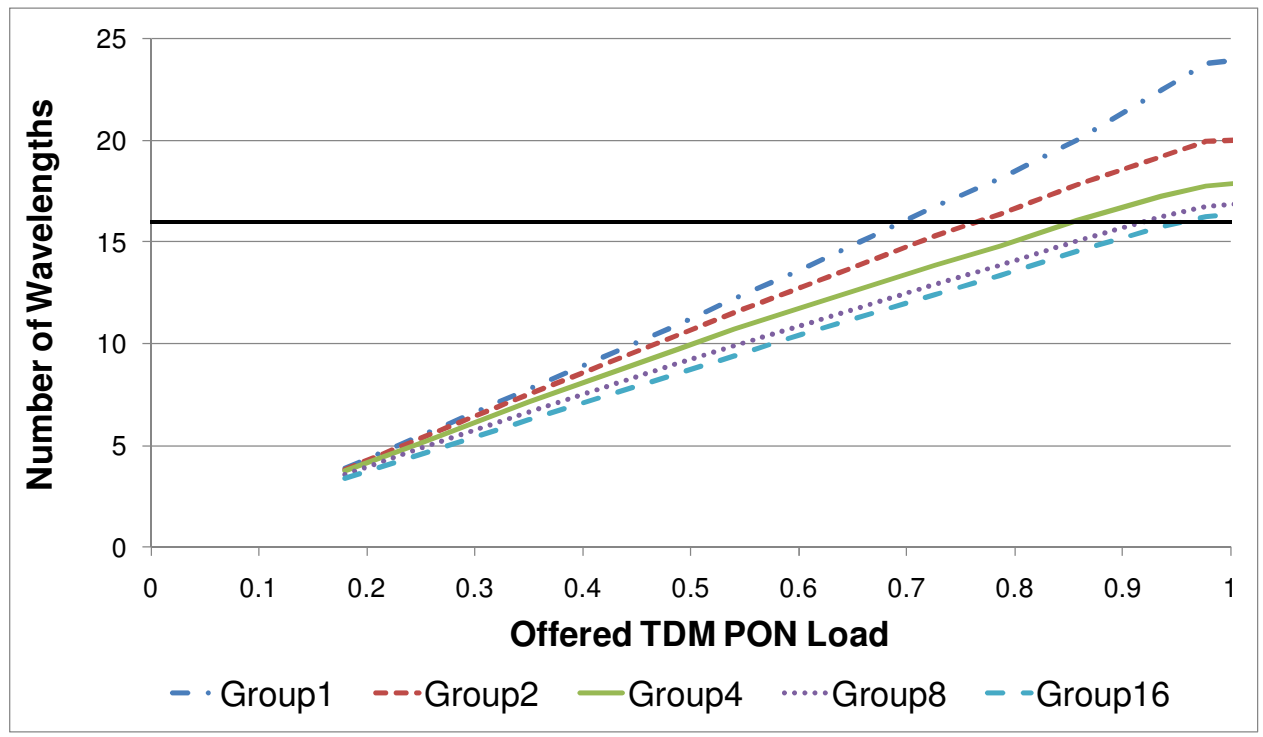

Figure 4: Average number of wavelengths required in function of the offered TDM PON or RN2 load, for five hybrid WDM/TDM PON (Group 1, 2, 4, 8, 16) with a different degree of flexibility in RN1

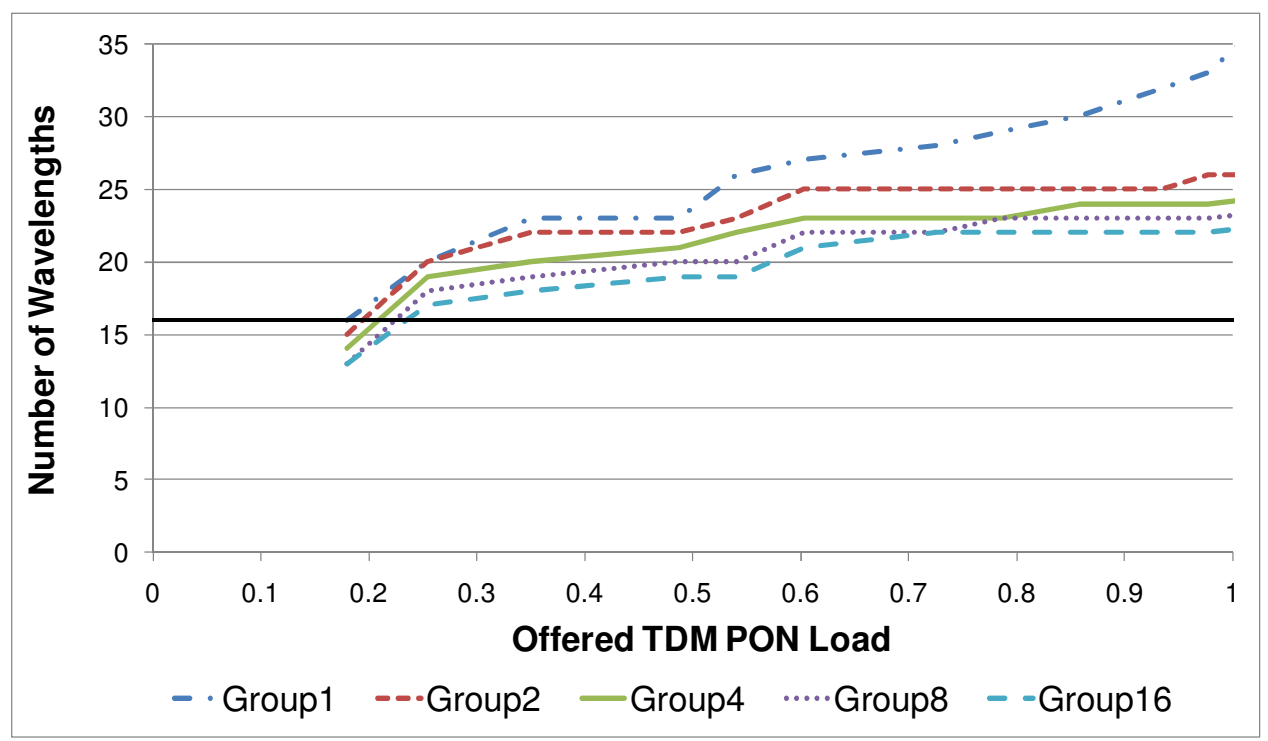

Figure 5: Maximum number of wavelengths required in function of the offered TDM PON or RN2 load, for five hybrid WDM/TDM PON (Group 1, 2, 4, 8, 16) with a different degree of flexibility in RN1

\section{Comparison between multicasting and switching flexibility}

In this section, we extensively compare the multicasting and switching flexibility. Figure 6 provides the histograms for the wavelength requirement for different network load conditions, using Group 4. Figure 6 contains six histogram plots for three different network load conditions (network load of 0.25, 0.55 and 0.95 ) and two different architectures (AWG-based (left) as well as WSS-based (right)). The histograms for the architectures with an AWG are 
the histograms of individual TDM PON groups as these architectures have a restriction on the maximum number of wavelengths that can be used by a certain group (e.g., four wavelengths for the Group 4 case). The histograms for the architectures with a WSS are the histograms for the overall network wavelength requirement as WSSs only have a restriction for the overall maximum number of required wavelengths by the entire network (i.e., 16 wavelengths for our simulation example) and do not impose any restriction for the individual groups. Note that all the simulations for the histogram plots are carried out without any restriction on the wavelength requirements for individual groups as well as the entire network. Therefore, in the simulations we allocate as many wavelengths as are requested by each individual group at a time. That is why almost all the histogram plots often have non-zero numbers even beyond the maximum number of wavelengths per group for the AWG-based architectures, and beyond the maximum number of wavelengths by the entire network for the WSS-based architectures. These non-zero figures indicate the probability of overloading, which is an important parameter to be considered further.
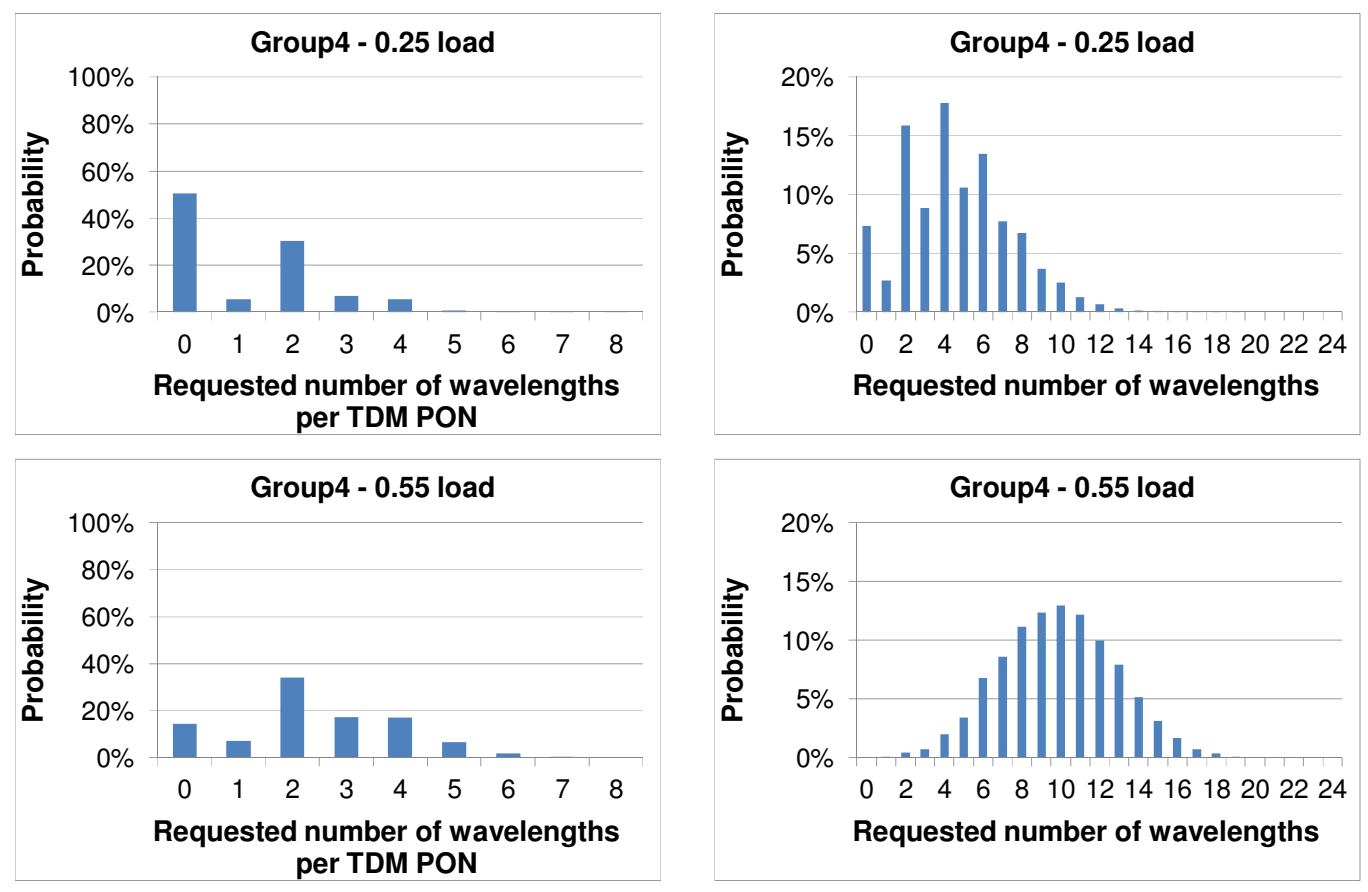

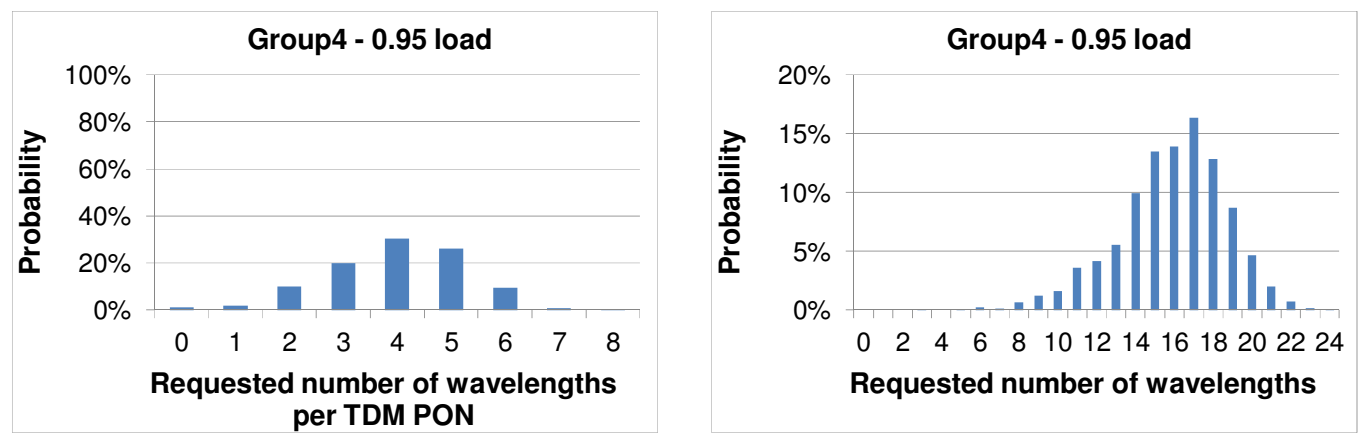

Figure 6: Histogram for the wavelength requirement, in case of Group 4 and highly bursty traffic

We calculate the network overload factor (NOF) as follows:

- For the AWG-based architectures with Group $m_{s}$ as shown in Figure 3(a), we calculate the cumulative probability of the group overload (e.g., the sum of the probability figures beyond $m_{s}$ from the histogram figures) as $P_{\text {overload_gr }}$. From this, we further calculate the overall NOF as follows:

NOF $=1-\left(1-\text { Proverload_gr }_{\text {r }}\right)^{M / m_{s}}$.

- For the WSS-based architectures, the NOF is just calculated as the cumulative probability of network overload (e.g., the sum of the probability figures beyond 16 wavelengths for the corresponding histogram figures).

The above described methodology in fact provides the flexibility evaluation basis for the two different architectures (as depicted in Figure 3(a) and (b)) from a single simulation scenario. In Figure 7 and 8 we compare these NOF figures between the two evaluated architectures (with AWG and with WSS, respectively) and for five different groups $(1,2,4,8,16)$, as function of the network load. We observe that the NOF figures increase as the load increases, as expected. Moreover, the NOF figures also increase as the group number decreases. This indicates the multicasting gain, attained thanks to the wavelength sharing among an increasing number of TDM PONs as the group number increases (i.e., an overall increment of the multicasting domain). 


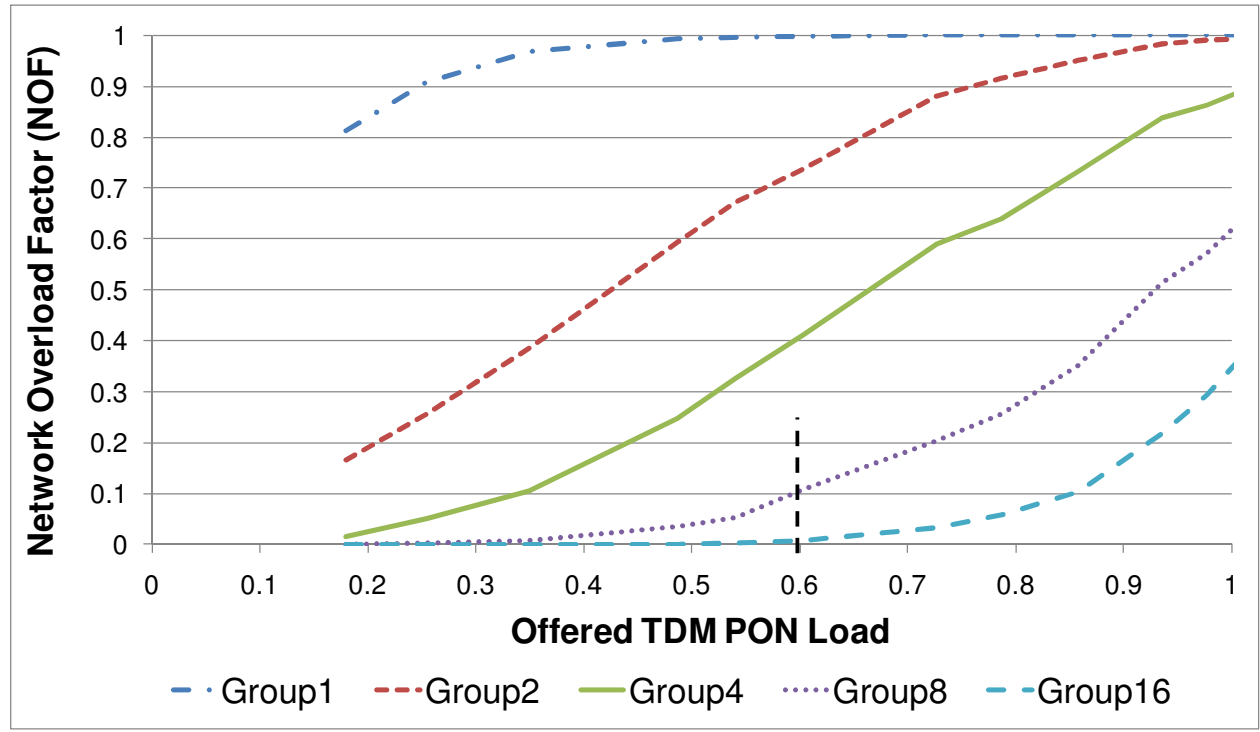

Figure 7: Network overload factor (NOF) for hybrid WDM/TDM PON architectures using AWGs and power splitters at a bursty traffic condition

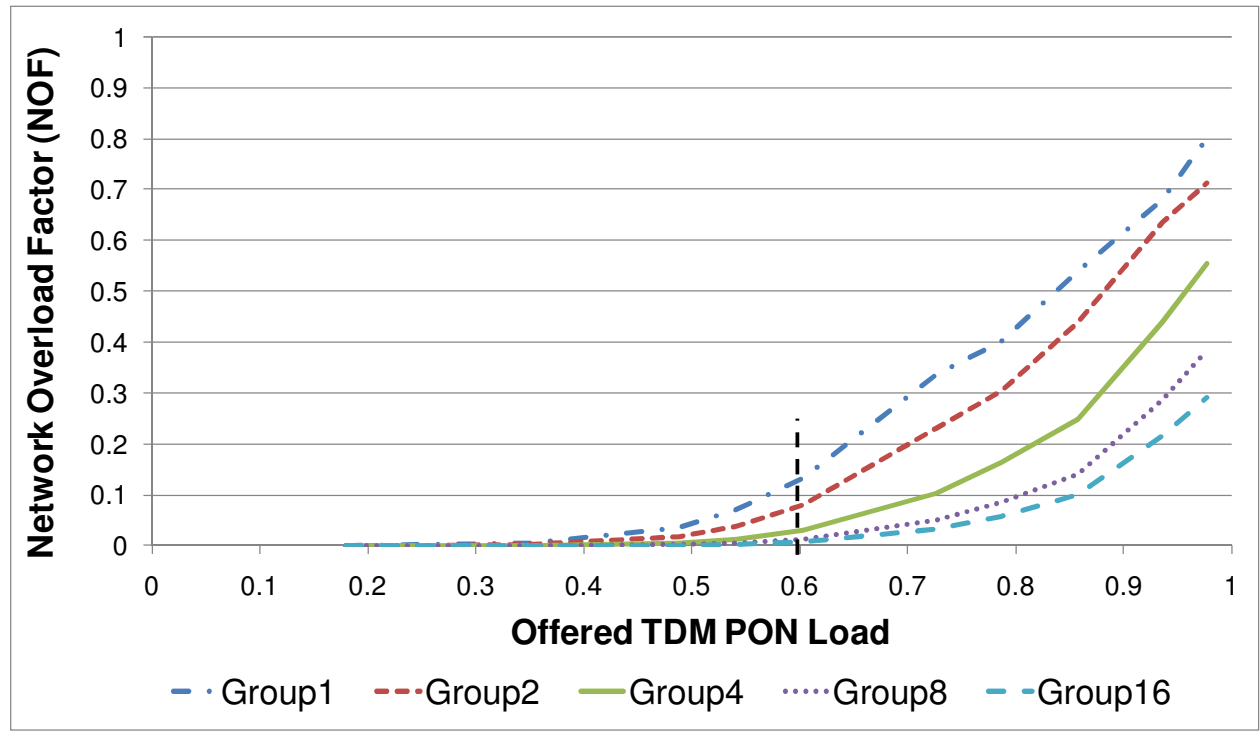

Figure 8: Network overload factor (NOF) for hybrid WDM/TDM PON architectures using WSSs and power splitters at a bursty traffic condition

We further observe that in Figure 8 compared to Figure 7, there are significant NOF figure differences among the corresponding AWG and WSS-based architectures (considering the same multicasting group). Therefore, we conclude that the architecture with WSSs reduces the NOF significantly and utilizes its wavelength resources much more efficiently. Now to quantify the benefit of using WSS and switching flexibility, we observe that at a TDM PON load 0.6, the architecture with AWG provides a NOF figure of 0.1 with a Group 8 
configuration whereas the architecture with WSS can already provide a better NOF figure of 0.08 with a Group 2 configuration. Therefore, for the same network load to achieve a similar network overload or network performance, we need a WSS combined with a 1:2 splitter compared to an AWG combined with a 1:8 splitter. This eventually provides an overall $7 \mathrm{~dB}$ power budget benefit (as each 1:2 split enforces almost $3.5 \mathrm{~dB}$ insertion loss).

From the above discussion we conclude that in general there exists a significant multicasting gain with increasing flexibility in the sense that more and more multicasting is allowed among different TDM PONs. However, the multicasting gain with partial flexibility (partial multicasting (Group 4 or 8) in contrast to total broadcasting (Group 16)) already offers large advantages, and often a full flexibility or total broadcasting is not needed. We further observe that as the traffic becomes more and more bursty, we attain a significant benefit of switching gain (using WSSs in place of AWGs) in a hybrid WDM/TDM PON. In section 6 we will provide numerical evidence that using switching devices (like a WSS) at the first remote node of a hybrid WDM/TDM PON does not affect the cost or power budget significantly. Although WSSs are not yet commonly used in the access network, this device is worthwhile to consider in more detail for next generation access networks, especially with highly bursty traffic patterns.

\section{Partially flexible architectures using WSS}

In the previous section we have already proven that WSS-based partially flexible architectures provide important flexibility benefits. In this section we therefore propose different variations of a WSS-based architecture. In all partially flexible architectures with a WSS, each TDM PON can be reached by multiple wavelengths. However, each wavelength can reach either some fixed number of TDM PONs or only one TDM PON or some variable numbers of selected TDM PONs. For that reason, we divide these architectures in three main categories: architectures with fixed multicasting, architectures without multicasting and architectures with selective multicasting, respectively. The architecture description and a depiction of all three variants are provided below.

\subsection{Partially flexible architectures, with fixed multicasting}

Two variants of a partially flexible hybrid WDM/TDM PON architecture with fixed multicasting using WSS, are discussed in this section (see Figure 9). In a partially flexible architecture with fixed multicasting, each wavelength can be routed to multiple predetermined TDM PONs (but not all TDM PONs can be reached by one wavelength at a time). 


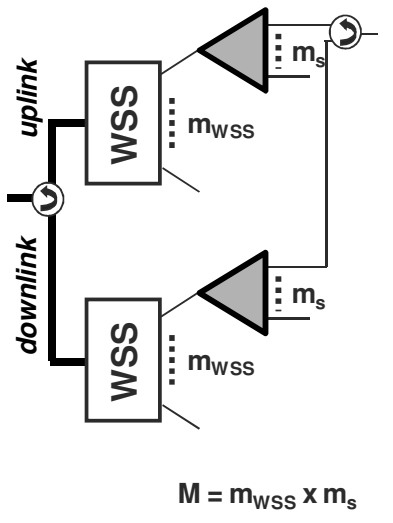

(a) Variant 1

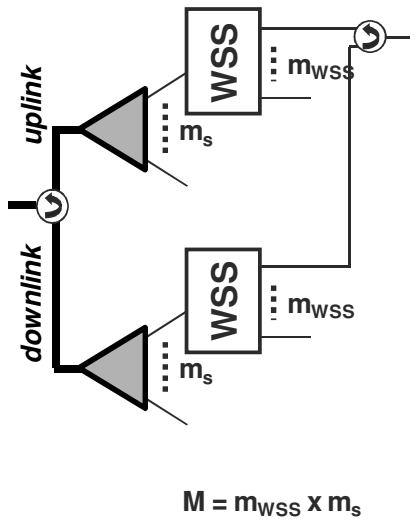

(b) Variant 2

Figure 9: Remote node 1 (RN1) variants for partially flexible hybrid WDM/TDM PON flavors with fixed multicasting

\section{Variant 1: B\&S hybrid WDM/TDM PON, power splitters combined with WSS}

In variant 1 of a B\&S hybrid WDM/TDM PON combined with WSS, RN1 consists of a wavelength selective switch (WSS) followed by multiple ( $m_{\text {WSS }}$ for the uplink and downlink direction, respectively) power splitters, as shown in Figure 9(a). In this architecture, the WSS works as a reconfigurable switch that can steer any wavelength to any of the power splitters. Note that this variant 1 of a WSS-based architecture has been extensively used and evaluated in the previous section for the flexibility analysis.

In contrast to an AWG used in a WS hybrid WDM/TDM PON, the wavelength to a particular output port is no longer static, but can be selected by the WSS, enhancing the flexibility of RN1. A WSS has the capability of steering one wavelength from an output port to another one if the users attached to the concerned output port do not require the service of that particular wavelength anymore. However, the multicasting group here is always fixed and is formed by all the TDM PONs connected to the same power splitter. Therefore, the number of users per multicasting group is always fixed and only the number of wavelengths shared among the same multicasting group can be variable. On the contrary, from the security perspective, there is not much difference between using an AWG and WSS. The main disadvantage of using a WSS, however, is its comparatively high cost, as discussed in section 6 .

\section{Variant 2: B\&S hybrid WDM/TDM PON, power splitters combined with WSS}

In variant 2 of a B\&S hybrid WDM/TDM PON combined with WSS, RN1 consists of a power splitter followed by multiple ( $m_{s}$ for the uplink and downlink direction, respectively) 
WSSs, as shown in Figure 9(b). In this architecture, the power splitter and WSS are changed from position.

As the power splitter is put in front of the WSS, the flexibility is further enhanced, as RN1 can now do a multicasting that is no longer limited to a fixed number of TDM PONs (i.e., the TDM PONs under the same multicasting group can be rearranged over time). However, the number of TDM PONs within the same multicast domain remains the same. For that reason, this variant 2 of a WSS-based architecture can as well be included within this multicasting category. The same wavelength can be multicast to $m_{s}$ different output ports of RN1, provided they are attached to different WSS modules. Here also, we can observe that the number of users per multicasting group remains the same. However, in this architecture, contrary to the previous one, different multicasting groups with different wavelengths can share common members. For example, if we assign wavelength $\lambda_{1}$ and $\lambda_{2}$ to port 1 and port 2 of the first WSS in Figure 9(b) and assign both $\lambda_{1}$ and $\lambda_{2}$ to port 1 of the next WSS, then users attached to the first port of the second WSS are the common members of two different multicasting groups assigned on wavelengths $\lambda_{1}$ and $\lambda_{2}$, respectively. The power budget and security aspects are comparable to the previous partially flexible architecture. The main disadvantage of this second variant of a WSS-based architecture, compared to the first variant, is its higher cost due to the use of a higher amount of WSSs $\left(m_{s}\right.$ instead of 1 for the uplink as well as the downlink direction).

\subsection{Partially flexible architectures, without multicasting}

Two variants of a partially flexible hybrid WDM/TDM PON architecture without multicasting, are discussed in this section (see Figure 10). In a partially flexible architecture without multicasting, each wavelength can be routed to one TDM PON only at a time (i.e., no multicasting of a wavelength among multiple TDM PONs is allowed). However, each TDM PON can still be reached by multiple wavelengths. In general, these architectures are extended WS-PONs, using a WSS as basic element. On the other hand, they do not make use of power splitters because no multicast functionality is needed, which means that their power budget is much better than for the partially flexible solutions with multicasting.

As multiple wavelengths can be offered to one TDM PON, there is still flexibility to adapt the number of wavelengths per TDM PON according to the traffic demand. However, since each wavelength can only be routed to one TDM PON simultaneously, this architecture is mainly of importance if the number of wavelengths $\left(W_{d}\right.$ and/or $\left.W_{u}\right)$ is larger than the number of TDM PONs $M$, because otherwise, e.g. if the number of wavelengths is equal to $M$, one or more 
TDM PONs cannot be reached from the moment another TDM PON is served by two or more wavelength.

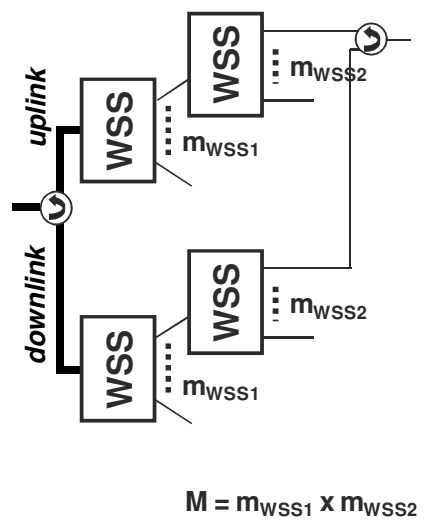

(a) Variant 3

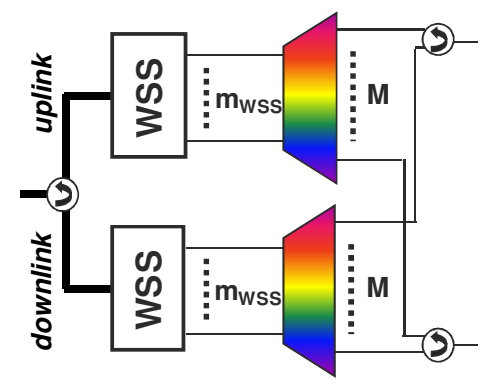

(b) Variant 4

Figure 10: Remote node 1 (RN1) variants for partially flexible hybrid WDM/TDM PON flavors without multicasting

\section{Variant 3: WS hybrid WDM/TDM PON, with WSS only}

In a WS-PON with WSS only (refer to Figure 10(a)), we have two stages of WSS interconnected to provide a fully reconfigurable architecture so that each of the available wavelengths can be steered to any output port. However, it does not provide any multicasting functionality as multiple output ports of RN1 cannot be reached by the same wavelength. This architecture is a costly solution; however, it provides the maximum switching gain. Note that multiple WSS stages are only required when the RN1 output port count increases beyond the maximum number of ports available per WSS (i.e. eight ports in the currently commercial systems).

\section{Variant 4: WS hybrid WDM/TDM PON, AWG extended with WSS}

In a WS-PON with AWG extended with WSS, RN1 consist of a WSS followed by an AWG, as shown in Figure 10(b). Compared to a standard WS-PON, a WSS enhances the wavelength reconfigurability among the subscribers according to their traffic demand. In particular, the WSS can select which wavelength to be routed to which input port of the AWG. Combing a WSS with an AWG provides partial flexibility of choosing multiple (with a maximum equal to the number of WSS output ports, i.e. $m_{W S S}$ ) wavelengths to be routed to a particular output port of the AWG.

As only a maximum of $m_{W S S}$ wavelengths can reach a particular TDM PON (or RN2), we can increase the flexibility of the proposed architecture by increasing the number of output ports 
per WSS. This brings a cost versus flexibility tradeoff issue in the architecture design. As for all WSS-based PONs, there is a costly WSS required, but just as in variant 1 of the WSSbased architectures, this solution only requires one WSS for upstream and downstream, respectively.

\subsection{Partially flexible architectures, with selective multicasting}

Next we introduce two architectural variants using a WSS to provide selective multicasting (see Figure 11). Note that this type of architectures is based on variant 4 of the WSS-based architectures presented in the previous subsection.

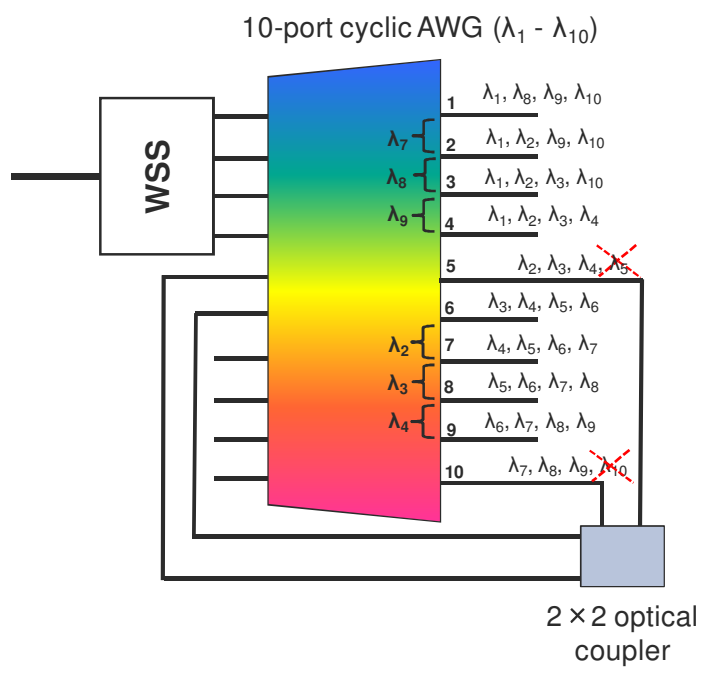

(a) Variant 5

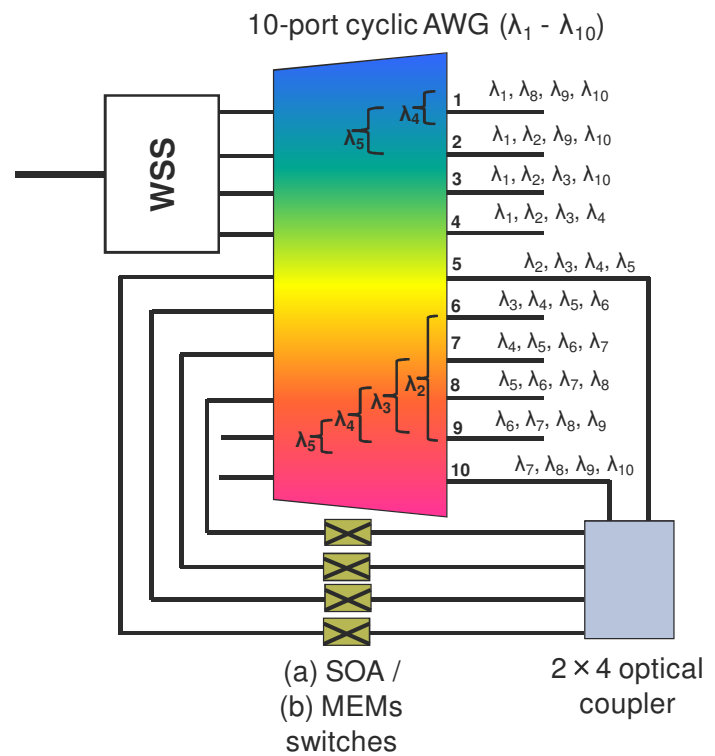

(b) Variant 6

Figure 11: Remote node 1 (RN1) variants for partially flexible hybrid WDM/TDM PON flavors with selective multicasting

Variant 5: WS hybrid WDM/TDM PON, AWG extended with WSS and feedback loop for selective multicasting

Figure 11(a) shows the RN1 part of such architecture which we will refer to as variant 5. For simplicity, we used only 8 fan-outs, corresponding to a 1:8 split of the RN1 (i.e., $M=8$ ). However, the fan-outs can be increased by simply increasing the number of ports per AWG and subsequently increasing the number of wavelengths. For our example, we have used a 10port cyclic AWG supporting 10 wavelengths. A 4-port WSS is used to switch the wavelengths $\left(\lambda_{1}\right.$ to $\left.\lambda_{10}\right)$ to one of its output ports according to the scheduled wavelength assignment for the connected TDM PONs. The AWG is used in the next stage to further distribute the 
wavelengths among the users. However, the combination of a WSS and AWG can only switch the entire wavelength from one output port to another. To increase the flexibility further, it is required to share wavelengths among multiple output ports (multicasting). For example, if port 1 and port 2 are used by business customers and residential customers respectively; then according to the hours of the day, the traffic demand shifts from one port to another. Therefore, sharing wavelengths among those two ports can further increase the possibility of statistical multiplexing and efficient resource utilization as the traffic demand pattern varies between hours. To enable this we introduce a $2 \times 2$ optical splitter that feeds the signal from two unused output ports of the AWG (e.g., port 5 and 10 in Figure 11(a)) to its two input ports (e.g., port 5 and 6 in Figure 11(a)). This facilitates the multicasting of wavelengths to two TDM PONs, as shown in Figure 11(a).

Figure 11(a) shows the possible combination of ports for wavelength multicasting and the corresponding wavelengths to be used. Figure 11(a) mentions the list of wavelengths that are allowed to be switched to each output port of the AWG. Now, as depicted in Figure 11(a), the wavelengths $\lambda_{2}, \lambda_{3}, \lambda_{4}, \lambda_{5}$ and $\lambda_{7}, \lambda_{8}, \lambda_{9}, \lambda_{10}$ can be switched (by WSS) to the $5^{\text {th }}$ and $10^{\text {th }}$ AWG output port, respectively. A careful consideration of the properties of a cyclic AWG will suggest that out of them $\lambda_{7}$ will be broadcast to port 1, 2 after passing the 1:2 splitter in the feedback loop (refer to Figure 11(a)). Similarly $\lambda_{8}$ will go to port 2,$3 ; \lambda_{9}$ will go to port 3 , $4 ; \lambda_{2}$ will go to port 6,$7 ; \lambda_{3}$ will go to port 7,$8 ; \lambda_{4}$ will go to port 8,9 . However, if we choose $\lambda_{5}$ or $\lambda_{10}$ they will be retransmitted to the same feedback path to cause interference with the existing signal. Therefore, during the selective multicasting configuration $\lambda_{5}$ or $\lambda_{10}$ are not allowed to be selected for output port5 and 10 of the AWG respectively (as indicated in Figure 11(a) with dashed crosses).

\section{Variant 6: WS hybrid WDM/TDM PON, AWG extended with WSS and switched feedback}

\section{loop for selective multicasting}

It is evident, that the flexibility can be further enhanced as the number of wavelengths as well as the number of output ports per WSS and AWG increases. However, using $2 \times 2$ optical splitters restricts us to share wavelengths among two output ports of the AWG. To enhance this feature further, we introduce the architecture shown in Figure 11(b).

Figure 11(b) enhances the multicasting capability of the architecture proposed in Figure 11(a) by introducing a $2 \times 4$ splitter and optical on-off switches to control the data flow path in the multicast domain. We refer to this architecture as variant 6 . For example, now if $\lambda_{2}$ is first directed to the output port of the AWG by the WSS it will further be multicast to port $6,7,8$ 
and 9. Now, due to the presence of the optical on/off switches, we can even control how many of the multicasting ports we prefer to allow. For our example we can prefer to multicast wavelength $\lambda_{2}$ to port 6 and 7 and consequently block it to go to port 8 and 9 by keeping the first two optical switches (connected to input port 7 and 8 of the AWG) in off state and the other two in on state. The switches additionally ensure that the wavelength retransmission as discussed for variant 6(a) never happens by blocking them using the optical switch. Though this architecture increases the flexibility, it comes with a cost of additional power budget due to the introduction of a higher splitter loss and switch loss in the feedback loop of the AWG. However, if we use semiconductor optical amplifiers (SOA) as switches (variant 6(a)), the SOA gain can compensate the additional insertion loss. However, SOA can introduce ASE noise. For our purpose, we can also use MEMS switches that are cost effective and easier to integrate (variant 6(b)). However, we need to keep in mind that the MEMS switches are comparatively slow and do not provide any gain.

\section{Evaluation of flexibility constraints}

As mentioned in section 3, introducing extra flexibility in the architecture will lead to some other architectural constraints, of which the equipment cost and power budget, influencing the reach, are the most critical ones. Note that the increased equipment costs will probably be compensated by the decreasing operational costs, as mentioned in section 3.2. However, quantifying the operational costs requires a detailed process modeling which is out of scope for this paper.

For the evaluations below, we have assumed three scenarios with a RN2 split $N=32$ and varying values for the RN1 split $M$ and the number of wavelength pairs $W_{u, d}$ at $10 \mathrm{Gbit} / \mathrm{s}$. The chosen values for $M, N$ and $W_{u, d}$, together with the resulting number of subscribers per OLT and the sustainable bandwidth per subscriber are summarized in Table 1 . The values for $M, N$ and $W_{u, d}$ are chosen in such a manner that the sustainable bandwidth per subscriber remains the same (i.e., $625 \mathrm{Mb} / \mathrm{s}$ ) across all scenarios.

Table 1: Considered scenarios for the equipment cost and power budget evaluation

\begin{tabular}{c||c||c|c|c|c} 
& $W_{u, d}$ & $\boldsymbol{M}$ & $\boldsymbol{N}$ & $\begin{array}{c}\text { \# subscribers } \\
\text { per OLT }\end{array}$ & $\begin{array}{c}\text { Sustainable } \\
\text { bandwidth }\end{array}$ \\
\hline \hline Scenario 1 & 64 & 32 & 32 & 1024 & $625 \mathrm{Mb} / \mathrm{s}$ \\
Scenario 2 & 32 & 16 & 32 & 512 & $625 \mathrm{Mb} / \mathrm{s}$ \\
Scenario 3 & 16 & 8 & 32 & 256 & $625 \mathrm{Mb} / \mathrm{s}$
\end{tabular}


As an addition to Table 1, we want to make two extra remarks. First of all, the partially flexible architectures without multicasting (variant 3 and 4) are only of importance if $W_{u, d}>M$ as then all the output ports of RN1 without multicasting can still have $\left(W_{u, d}-M\right)$ additional wavelengths left for flexibility even after allocating one mandatory wavelength to each output port. Otherwise the flexibility gain due to switching will have no significance at all. This is the reason why we have always chosen values for $W_{u, d}$ greater than $M$ in all our scenarios. Secondly, for the partially flexible architectures with fixed multicasting, there is an extra design parameter in RN1 as the 1:M split is done in two different stages (see Figure 9). Here, we assume that the splitting ratio of the WSS (resp. $m_{W S S}$ ) is always equal to 8 , except for the case where $M=8$. There as the $m_{W S S}$ must always be less than $M$ we have chosen the value of $m_{W S S}$ as 4 .

\subsection{Equipment cost}

In this section, two equipment cost evaluation are performed. First, for the three scenarios presented in Table 1, we compare the equipment cost between the nine different hybrid WDM/TDM PON architectures discussed in section 1 and 5: B\&S-PON (i.e. fully flexible), WS-PON (i.e. fully static), and the seven proposed partially flexible architectures using a WSS. Secondly, a more detailed evaluation is made for the different partially flexible hybrid WDM/TDM PON architectural options.

Table 2 gives an overview of the assumed cost figures of the most important components used in the remote nodes of the different hybrid WDM/TDM PON architectures, and leading to the cost differentiation of the different architectures. Note that we assume a cost of $4 €$ per port of a passive power splitter (leading to a cost of $12 €$ for a 1:2 splitter, $20 €$ for a 1:4 splitter, etc.). For 16 customers per wavelength, we assume an OLT cost of ca. $400 €$ per customer. Finally, for the ONU we assume a cost of $500 €$. All these costs correspond to reasonable target costs for within 5 years, when the first hybrid WDM/TDM PONs will probably enter the market.

Table 2: Overview of costs of the optical components used in the remote nodes of the considered hybrid WDM/TDM PONs

\begin{tabular}{l|l||l|l}
\multicolumn{1}{c|}{ Component } & \multicolumn{1}{c|}{ Cost } & \multicolumn{1}{c}{ Component } & \multicolumn{1}{c}{ Cost } \\
\hline \hline Power splitter & $4 €($ per port) & $1 \times 2 \mathrm{WSS}$ & $4500 €$ \\
AWG & $500 €$ & $1 \times 4 \mathrm{WSS}$ & $7500 €$ \\
& & $1 \times 8 \mathrm{WSS}$ & $12500 €$
\end{tabular}


In Figure 12, the cost per subscriber is depicted for the three scenarios and the nine mentioned architectures, and a split is made between the cost for the ONU, the remote nodes (both RN1 and RN2) and the OLT. Note that we ignore the cost for fiber installation as this cost remains identical for each architecture within a certain scenario. A full cost analysis, including fiber installation, for some hybrid WDM/TDM PON architectures is given in [17].

When evaluating the cost per subscriber for each of the proposed architectures, the main conclusion is that due to the sharing of RN1 among multiple users (i.e. 256, 512 and 1024 in the examples of Figure 12), the cost for reconfigurability does minimally alter the overall cost per subscriber in most of the described scenarios. This opens good perspectives for advanced flexible hybrid WDM/TDM PON technologies that will probably lower the operational costs. We also clearly notice that the larger sharing ratio (1024 subscribers) for scenario 1 results in a lower cost per subscriber. However, for a few architectures, like the one requiring more than two WSSs (e.g., variant 2 and 3) the overall cost is comparatively higher.

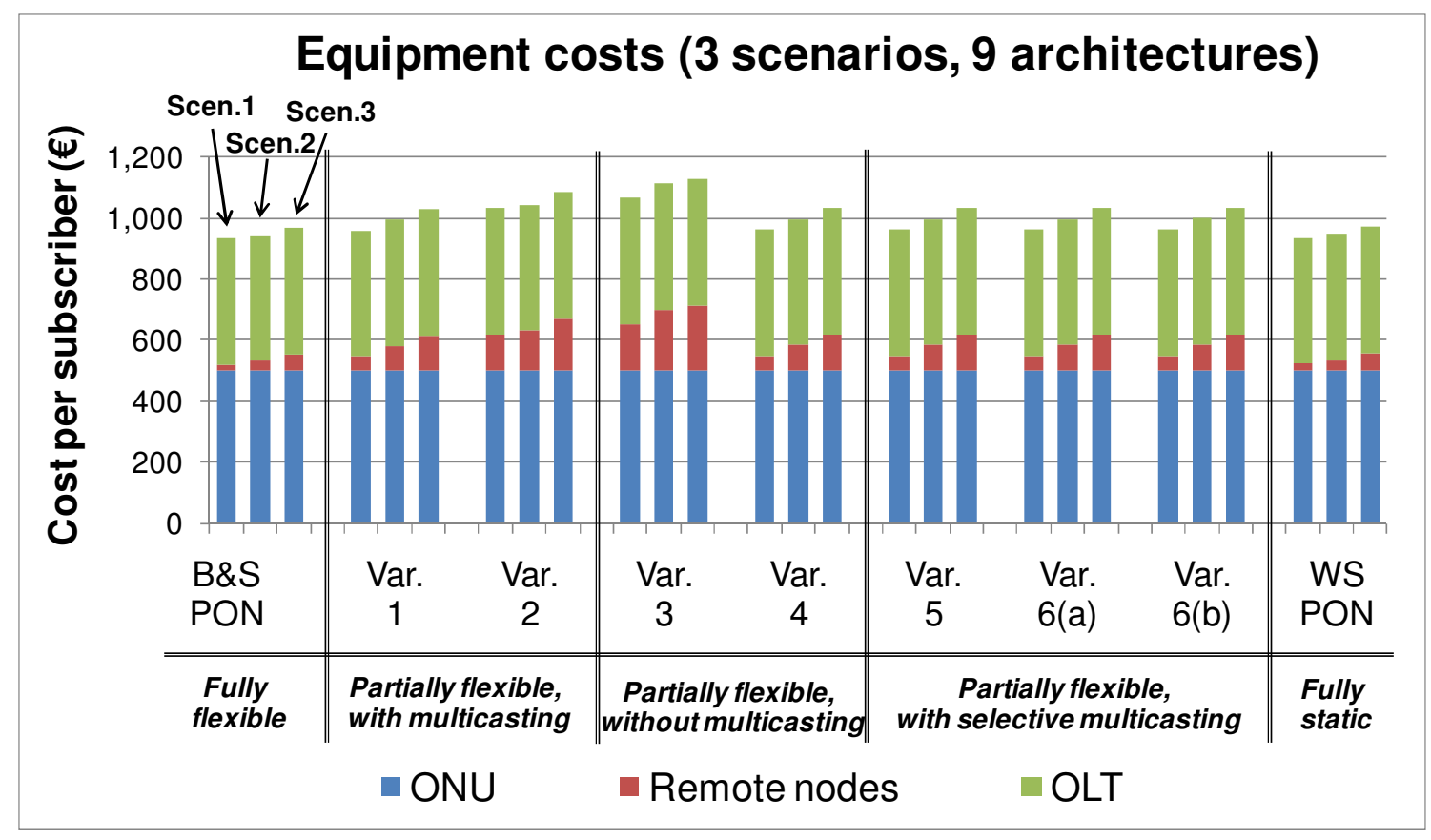

Figure 12: Cost breakdown for different WDM/TDM PON architectures

In a second evaluation, we further evaluate the relative difference of the cost figures due to the choice of $m_{W S S}$ in RN1. Here we evaluate only scenario 1 as this provides the most cost effective solution and ensures a higher degree of node consolidation. As varying $m_{W S S}$ does not alter the cost figures of the OLT and ONU, we concentrate on the overall remote node cost (i.e., the combined cost of RN1 and RN2). Figure 13 shows the remote nodes costs for 
the different variants of a partially flexible architecture using a WSS. For each variant, we have taken three values of $m_{W S S}=8,4,2$ respectively. For variant 3 as $m_{W S S}=2$ means that the second stage of RN1 will require a WSS with 16 ports, we have ignored this possibility due to the commercial unavailability of a 16-port WSS.

We observe that except for variant 2 and 3 as mentioned earlier, none other variant imposes a significant cost increment related to the two benchmark passive architectures (e.g., B\&S-PON and WS-PON). Therefore we conclude that adding flexibility to the first remote node does not significantly alter the total capital expenditure of an optical access network deployment.

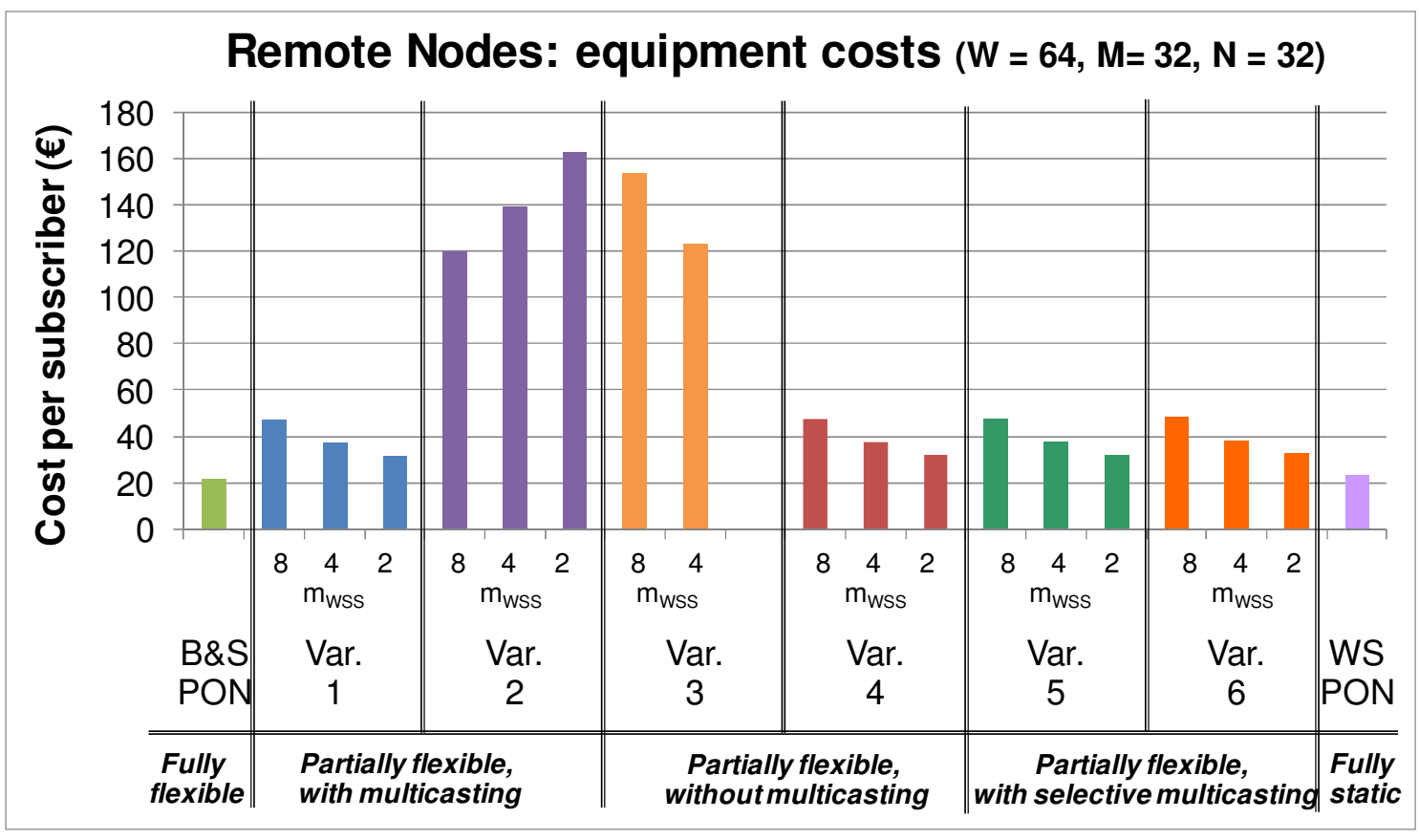

Figure 13: Remote nodes costs for different partially flexible hybrid WDM/TDM PON architectures, with multicasting (and compared to B\&S-PON and WS-PON).

\subsection{Power budget and reach}

In this section we calculate the power budget and the optical reach possible for the nine considered hybrid WDM/TDM PON architectures. Table 3 gives an overview of the assumed insertion losses of the most important components used in the different hybrid WDM/TDM PON architectures. Note that we assume a $3.5 \mathrm{~dB}$ loss per 1:2 power split instead of the theoretical loss of $3 \mathrm{~dB}$, as the latter is never attained in real implementations. For the AWG we assume a channel spacing of $100 \mathrm{GHz}$ which is sufficient for a maximum of 32 wavelengths per direction. For AWGs with a $50 \mathrm{GHz}$ spacing, however, the insertion loss will 
increase with roughly $1 \mathrm{~dB}$. Further, an optical component can be coupled by using a connector (ca. $0.5 \mathrm{~dB}$ ) or a splice (ca. $0.1 \mathrm{~dB}$ ). In our calculations, we use an average connector loss of $0.25 \mathrm{~dB}$.

Table 3: Overview of insertion losses of the optical components used in the considered hybrid WDM/TDM PONs

\begin{tabular}{l|l||l|l}
\multicolumn{1}{c|}{ Component } & Insertion loss $(\mathbf{d B})$ & Component & Insertion loss $(\mathbf{d B})$ \\
\hline \hline 1:2-splitter & 3.5 & Connector/splice & 0.25 \\
AWG & 3 & 3-port circulator & 0.25 \\
WSS & 4 & Optical filter & 2.5
\end{tabular}

Table 4 summarizes the maximum optical reach possible for the scenarios from Table 1. Moreover, two additional scenarios of 512 users (i.e., $M=32$ and $N=16$ ) and 256 users (i.e., $M=16$ and $N=16$ ), and with a sustainable bandwidth per subscriber of $625 \mathrm{Mb} / \mathrm{s}$, have been introduced to explore the relative benefits of the different architectures even more rigorously. We assume a fiber loss of $0.3 \mathrm{~dB} / \mathrm{km}$, a Tx power of $3 \mathrm{dBm}$, a receiver sensitivity of $-27 \mathrm{dBm}$ (ordinary PD) and a power penalty of $2 \mathrm{~dB}$. To support a longer reach, we also include an erbium-doped fiber amplifier (EDFA) in RN1 (e.g., an amplifier stage in the street cabinet), with a gain of $25 \mathrm{~dB}$ in both the uplink and downlink direction. Additionally the SOA switch gain is assumed to be $12 \mathrm{~dB}$ for architecture variant $6(\mathrm{~b})$.

From Table 4, it is clear that a WS-PON is the best option from a power budget perspective, but it has no flexibility in terms of wavelength switching. In section 4 , it was shown that most of the time full flexibility is not required, and only a partially flexible architecture can already be of great advantage. In this way, the partially flexible architectures come into the picture. If multicasting is introduced, the power budget and reach is somewhat lowered, but in a very limited way. These partially flexible architectures using WSS and multicasting will probably be of great importance for designing next generation optical access architectures, as they have a satisfactory optical reach, very negligible cost hike and a reasonable amount of flexibility. Only variant 2 and 3 will probably be too costly (as indicated in Figure 12) for arguing in favor of these architectures. 
Table 4: Reach of different hybrid WDM/TDM PONs

\begin{tabular}{|c|c|c|c|c|c|}
\hline $\begin{array}{c}\text { PON } \\
\text { architecture }\end{array}$ & $\begin{array}{c}\text { Reach }(\mathrm{km}) \text {, } \\
1024 \text { users } \\
(32 \times 32)\end{array}$ & $\begin{array}{c}\text { Reach }(\mathrm{km}) \text {, } \\
512 \text { users } \\
(16 \times 32)\end{array}$ & $\begin{array}{c}\text { Reach }(\mathrm{km}), \\
512 \text { users } \\
(32 \times 16)\end{array}$ & $\begin{array}{c}\text { Reach }(\mathrm{km}), \\
256 \text { users } \\
(8 \times 32)\end{array}$ & $\begin{array}{c}\text { Reach }(\mathrm{km}), \\
256 \text { users } \\
(16 \times 16)\end{array}$ \\
\hline B\&S-PON & 28 & 40 & 40 & 52 & 52 \\
\hline WS-PON & 72 & 72 & 83 & 72 & 83 \\
\hline Variant 1 & 43 & 55 & 55 & 67 & 67 \\
\hline Variant 2 & 43 & 55 & 55 & 67 & 67 \\
\hline Variant 3 & 53 & 53 & 65 & 53 & 65 \\
\hline Variant 4 & 57 & 57 & 68 & 57 & 68 \\
\hline Variant 5 & 42 & 42 & 53 & 42 & 53 \\
\hline Variant 6(a) & 27 & 27 & 38 & 27 & 38 \\
\hline Variant 6(b) & 70 & 70 & 82 & 70 & 82 \\
\hline
\end{tabular}

\section{Conclusion}

In this paper we have proposed different variants of partially flexible architectures using WSS as a key component. We have further introduced the definition of flexibility and categorized the flexibility gain in a PON scenario into two major categories; (a) multicasting flexibility gain generally provided by the power splitter and (b) switching flexibility gain provided by optical switches like a WSS. Through simulation we have proven that the partial replacement of multicasting gain with switching gain can often provide important architectural benefits in terms of reduced power budget, longer reach or higher degree of node consolidation without compromising the flexibility at all. Finally, we have shown through a cost and power budget calculation that some of our proposed architectures with WSS provide comparable cost figures to one of the cheapest hybrid WDM/TDM PON solutions present in the literature (B\&S hybrid PON) and comparable power budget figures to one of the most power budget efficient hybrid WDM/TDM PON solutions present in the literature (WS hybrid PON). The WSS-based hybrid WDM/TDM PON solutions, however, still need further research in a laboratory environment before they can be considered for commercial use. Moreover, the flexibility advantages, like network extensibility, energy efficiency and network migration, should be studied in more detail to quantify their importance in terms of improved network performance as well as reduced operational costs. 


\section{Acknowledgements}

The research leading to these results has received funding from the European Community's Seventh Framework Programme (FP7/2007-2013) under grant agreements nº 212352 (ICTALPHA) and $n^{\circ} 249025$ (ICT-OASE).

\section{References}

[1] IEEE 802.3ah task force home page [Online]. Available: http://www.ieee802.org/3/efm.

[2] ITU-T G.984.x series of recommendations [Online]. Available: http://www.itu.int/rec/T-REC-G/e.

[3] T. Koonen, "Fibre to the Home/Fibre to the Premises: what, where, and when?", Proc. of the IEEE, Vol. 94, No. 5, May 2006, pp. 911-934.

[4] C. Bock, J. Prat, and S. D. Walker, "Hybrid WDM/TDM PON Using the AWG FSR and Featuring Centralized Light Generation and Dynamic Bandwidth Allocation”, Journal of Lightwave Technology, Vol. 23, No. 12, Dec. 2005, pp. 3981-3988.

[5] N. Calabretta, M. Presi, R. Proietti, G. Contestabile, and E. Ciaramella, "A Bidirectional WDM/TDM-PON Using DPSK Downstream Signals and a Narrowband AWG”, IEEE Photonics Technology Letters, Vol. 19, No. 16, Aug. 2007, pp. 1227-1229.

[6] D. Breuer, F. Geilhardt, R. Hülsermann, M. Kind, C. Lange, T. Monath, and E. Weis, "Opportunities for Next Generation Optical Access”, IEEE Communications Magazine, Vol. 49, No. 2, Feb. 2011, pp. s16-s24.

[7] N.C. Tran, H.D. Jung, C. Okonkwo, E. Tangdiongga, and T. Koonen, "ARON: A SOA Array-based WDMTDM Reconfigurable Optical Access Network”, Future Network \& Mobile Summit 2010, Florence, Italy, Jun. 16 - 18, 2010.

[8] H.D. Jung, N.C. Tran, E. Tangdiongga, and T. Koonen, "New Architecture for reconfigurable WDM-PON Networks based on SOA Gating Array", 9th International Conference on Optical Internet (COIN), Korea, Jul. 11 - 14, 2010.

[9] G. Das, B. Lannoo, H.-D. Jung, T. Koonen, D. Colle, M. Pickavet, and P. Demeester, "A New Architecture and MAC Protocol for Fully Flexible Hybrid WDM/TDM PON”, Proc. of ECOC 2009, Vienna, Austria, Sep. 20 - 24, 2009, paper P6.28.

[10] G. Das, B. Lannoo, D. Colle, M. Pickavet, and P. Demeester, "A Hybrid WDM/TDM PON Architecture Using Wavelength Selective Switches", Proc. of ANTS 2010, Mumbai, India, Dec. 16 - 18, 2010.

[11]R. Jensen, and A. Lord, "Novel Non-Blocking Low Loss Scalable WSS Architecture", Proc. of OFC/NFOEC 2008, San Diego, USA, Feb. 24 - 28, 2008, paper OthA6.

[12] W.E Leland, M.S. Taqqu, W. Willinger, and D.V. Wilson, "On the self-similar nature of Ethernet traffic", IEEE/ACM Transactions on Networking, Vol. 2, No. 1, Feb. 1994, pp. 1-15.

[13] W. Willinger, M. Taqqu, R. Sherman, and D. Wilson. "Self-similarity through highvariability: statistical analysis of Ethernet LAN traffic at the source level", Proc. of ACM SIGCOMM '95, Cambridge, USA, Aug. 1995, pp. 100-113.

[14] M. P. McGarry, and M. Reisslein, "Bandwidth management for WDM EPONS", Journal of Optical Networking, Vol. 5, No. 9, Sep. 2006, pp. 637 - 654. 
[15] A. R. Dhaini, C. M. Assi, M. Maier, and A. Shami, "Dynamic wavelength and bandwidth allocation in hybrid TDM/WDM EPON networks", Journal of Lightwave Technology, Vol. 25, No. 1, Jan. 2007, pp. 277 $-286$.

[16] M. P. McGarry, M. Reisslein, C. J. Colbourn, M. Maier, F. Aurzada, and M. Scheutzow, "Just-in-time scheduling for multichannel EPONs", Journal of Lightwave Technology, Vol. 26, No. 10, May 2008, pp. 1204-1216.

[17] B. Lannoo, G. Das, M. De Groote, D. Colle, M. Pickavet, and P. Demeester, "Techno-economic feasibility study of different WDM/TDM PON architectures", Proc. of ICTON 2010, 12th International Conference on Transparent Optical Networks, Munich, Germany, Jun. 27 - Jul. 1, 2010. 